\title{
Ultramicrotomy of Various Glasses and Glass-Corrosion Interfaces
}

\author{
KD Derr, Andrew C. Buechele, Cavin T. F. Mooers, and Ian L. Pegg \\ Vitreous State Laboratory, The Catholic University of America, Washington, D.C. 20064
}

A synthesis of protocols, from multiple disciplines, was utilized to prepare many types of glasses for transmission electron microscopy (TEM.) These types included single phase, amorphous-amorphous phase-separated, and aqueous corroded glasses, as well as glasses containing secondary crystalline phases. Samples to be ultramicrotomed were strategically cleaved, oriented, embedded, and sectioned. Relatively site specific regions were attained from glass samples by cleaving with an ultra fine-tipped carbide probe beneath a stereomicroscope, resulting in a fragment size between 20 and 50 microns. Fragments were treated with $\mathrm{Z} 6040^{\mathrm{TM}}$, which promotes particle adhesion to the resin matrix by forming a network of polymer chains around the particle [1,2]. These fragments were embedded in a hard Spurr's mixture on Aclar ${ }^{\mathrm{TM}}$ embedding film and vacuum infiltrated. After curing, the fragments were removed with a razorblade beneath a stereomicroscope. The fragment-inresin-matrix was carefully carved to make a stable fit in the desired orientation in a conical tip BEEM $^{\mathrm{TM}}$ capsule and so inserted. The BEEM ${ }^{\mathrm{TM}}$ capsule was then filled with the same hard Spurr's formula and cured. A single parallel cut was made into the block face to create a leading edge, and facing off was conducted with a fixed razor blade. Sectioning was conducted utilizing 35 and 45 degree diamond knives. As is typical with the sectioning of very hard and brittle materials, the glass cleaves at the knife edge, rather than being cut smoothly, forming a series of plate-like segments within the resin parallel to the cutting direction. The ability to routinely section glasses makes extensive TEM studies of such materials much more accessible than the traditionally labor intensive and comparatively less successful methods of dimpling and ion milling, or wedge polishing. In the case of corroded glasses, ultramicrotomy also enables much greater lengths of the corroded interface to be quickly prepared than is possible using alternate methods. Artifacts commonly introduced by ion milling are also avoided.

The Vitreous State Laboratory routinely tests many glass formulations for durability against aqueous corrosion by a variety of test protocols[3]. A modified layer develops on the surface of the glass under test as the glass network breaks down and components of the glass are released into solution. Figure 1 displays four micrographs of ultramicrotomed specimens of a high-sodium borosilicate glass showing the reaction interface along with some of the leached layer that develops in a procedure known as the Vapor Hydration Test (VHT.) Careful study of this interface in this and other glasses should lead to a better understanding of the mechanisms operative in glass corrosion and to the production of more durable glasses. Micrographs were taken with a JEOL JEM2000FX TEM at $200 \mathrm{KV}$.

\section{References}

[1] Lindley, V.A., Microsc. Res. \& Techn., 21, 355-360, (1992).

[2] Swab, Phil, Microsc. Res. \& Techn., 31, 308-310, (1995).

[3] Feng, X., et al, Ceramic Transactions, 45, 243-254, (1994). 


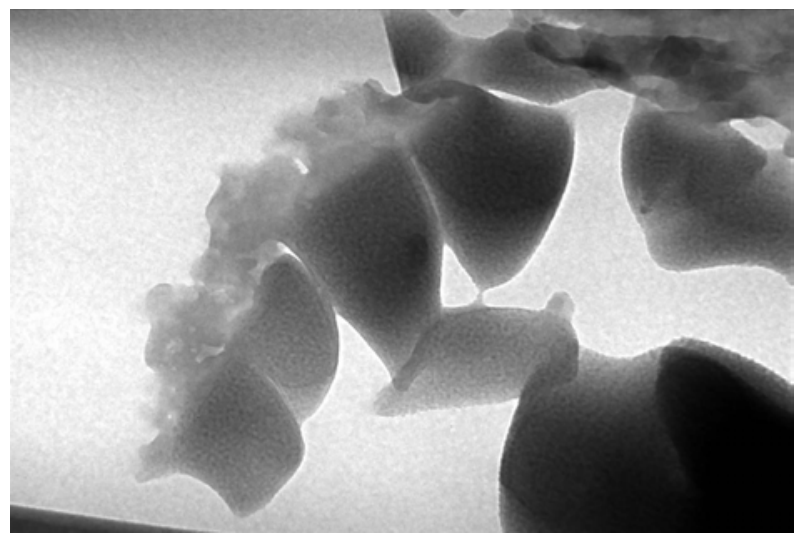

a)

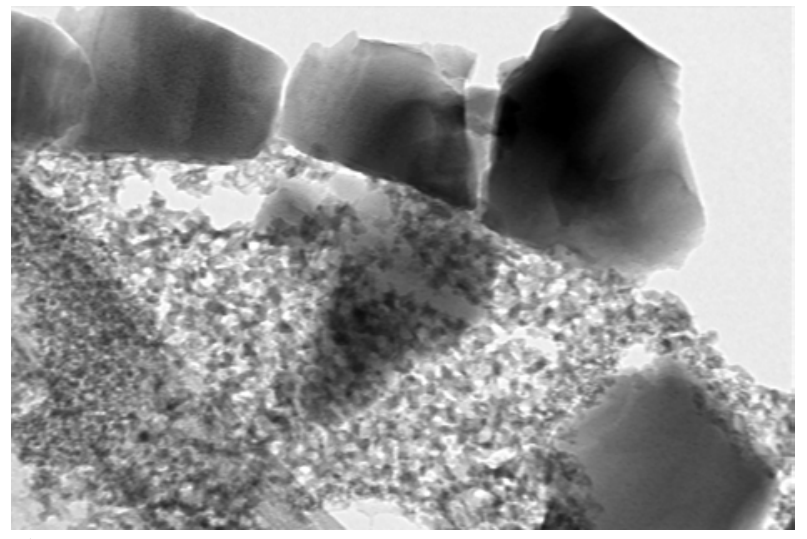

c)

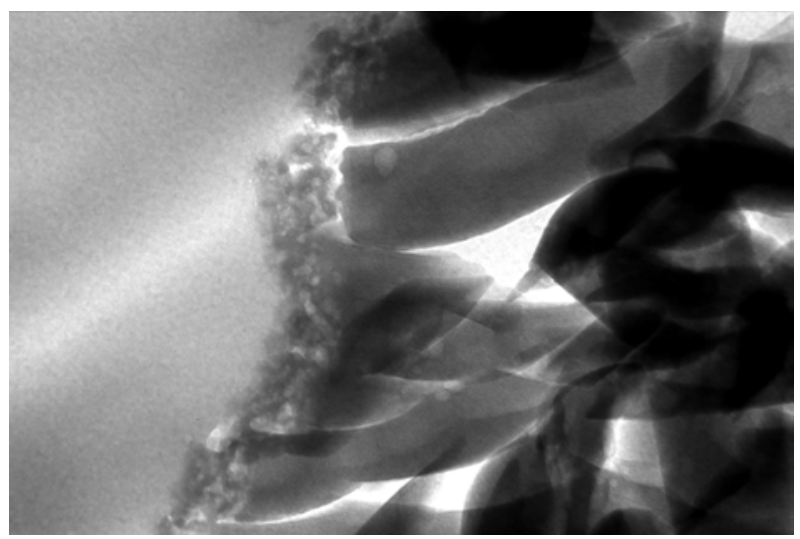

b)

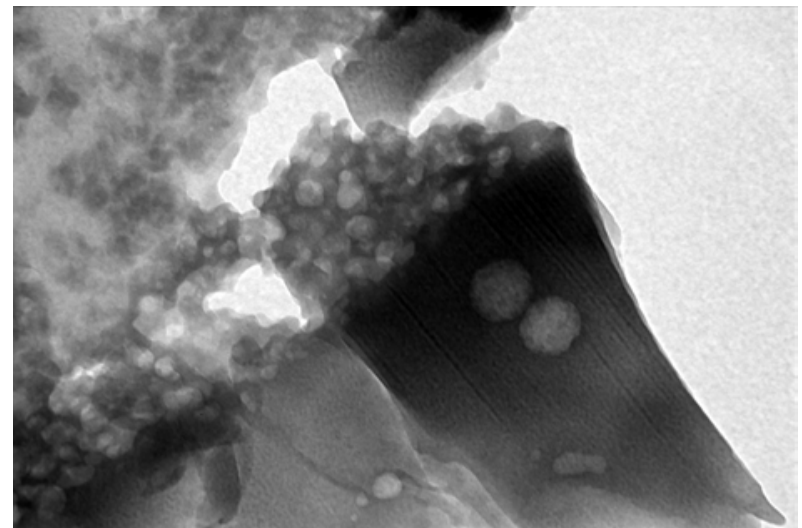

d)

FIG. 1 Sectioned VHT corroded glass with attached leached layer. a) 100K, b) 80K, c) 80K, d)120K. 